\title{
Video Article \\ Dissection and Downstream Analysis of Zebra Finch Embryos at Early Stages of Development
}

\author{
Jessica R. Murray ${ }^{1}$, Monika E. Stanciauskas ${ }^{1}$, Tejas S. Aralere ${ }^{1}$, Margaret S. Saha ${ }^{1}$ \\ ${ }^{1}$ Department of Biology, College of William and Mary
}

Correspondence to: Margaret S. Saha at mssaha@wm.edu

URL: https://www.jove.com/video/51596

DOI: doi:10.3791/51596

Keywords: Developmental Biology, Issue 88, zebra finch (Taeniopygiaguttata), dissection, embryo, development, in situ hybridization, 5-ethynyl-2'deoxyuridine (EdU)

Date Published: 6/21/2014

Citation: Murray, J.R., Stanciauskas, M.E., Aralere, T.S., Saha, M.S. Dissection and Downstream Analysis of Zebra Finch Embryos at Early Stages of Development. J. Vis. Exp. (88), e51596, doi:10.3791/51596 (2014).

\section{Abstract}

The zebra finch (Taeniopygiaguttata) has become an increasingly important model organism in many areas of research including toxicology ${ }^{1,2}$, behavior $^{3}$, and memory and learning ${ }^{4,5,6}$. As the only songbird with a sequenced genome, the zebra finch has great potential for use in developmental studies; however, the early stages of zebra finch development have not been well studied. Lack of research in zebra finch development can be attributed to the difficulty of dissecting the small egg and embryo. The following dissection method minimizes embryonic tissue damage, which allows for investigation of morphology and gene expression at all stages of embryonic development. This permits both bright field and fluorescence quality imaging of embryos, use in molecular procedures such as in situ hybridization (ISH), cell proliferation assays, and RNA extraction for quantitative assays such as quantitative real-time PCR (qtRT-PCR). This technique allows investigators to study early stages of development that were previously difficult to access.

\section{Video Link}

The video component of this article can be found at https://www.jove.com/video/51596/

\section{Introduction}

The overall goal of this technique is to obtain zebra finch (Taeniopygiaguttata) embryos from the earliest stages of embryogenesis for use in a wide range of developmental studies. Zebra finch have become the predominant songbird model organism and have been used extensively in a variety of fields, including toxicology ${ }^{1,2}$, ,ehavior $^{3}$, memory and learning ${ }^{4,5,6}$, comparative neuroanatomy ${ }^{7,8}$, and language development ${ }^{9,16}$. As the only songbird with a sequenced genome, the zebra finch allows molecular and genetic study of the Passeriformes order, which represents over $50 \%$ of known bird species ${ }^{11,12,13}$.

Despite the use of adult and juvenile zebra finch in a diverse array of fields, few studies have been performed on zebra finch embryos, particularly during early stages of development. This can be attributed to the small size of their eggs and embryos, and their newer status as a model organism ${ }^{14,15,16}$ for studies in which the chicken (Gallus gallusdomesticus) was previously used as a predominant model system $^{17,18,19,20,21}$. However, as non-vocal learners, chickens are not an appropriate model system for studying the genetic basis of vocal learning, development of vocal learning, heritability, behavior, and the cortical-basal ganglia circuitry involved in motor learning ${ }^{10}$.

It is important to note that zebra finch embryos are much more delicate and more easily damaged than chick embryos during dissection and molecular procedures. In particular, greater care is required when performing permeabilization steps on zebra finch embryos. Strong detergents and enzymes that would not harm a chick embryo can damage zebra finch embryos. In terms of general care, it is necessary to put zebra finch eggs in small cups before placement in an incubator to prevent them from breaking when rolling during incubation.

Zebra finch are amenable to behavioral studies, easily and prolifically breed year-round in captivity, and are vocal learners. These characteristics allow the use of zebra finch to address the need for a model organism that integrates development, genetics, and behavioral aspects of language. The dissections methods detailed below, combined with a recently developed staging guide specific to zebra finch ${ }^{22}$, make the zebra finch an increasingly useful standardized developmental model organism. However, obtaining embryos at early stages can be daunting. This protocol allows investigators to easily obtain early stage embryos. Studies investigating the early development and the molecular developmental basis of complex behaviors in zebra finch, or the toxicological effects on development in other small, passerine birds will find this dissection methodology useful.

\section{Protocol}

Ethics Statement: The methods were conducted with domesticated zebra finches from the breeding colony at the College of William and Mary. All procedures followed RSPCA guidelines ${ }^{23}$ and were approved by the College of William and Mary's OLAW (Office of Laboratory Animal 
Welfare) Animal Welfare Assurance (\#A3713-01) and had Institutional Animal Care and Use Committee (IACUC) approval (\#2013-06-02-8721dacris).

\section{Egg Collection and Incubation}

1. Establish zebra finch pairs on a 14:10 light: dark cycle. Supply food, water, hay, and a nest box ad libitum. Note: Routine care and breeding for zebra finches has been well described ${ }^{23}$.

2. Prepare a standard chick incubator with tilting shelves. Note: Maintain the artificial incubator at $37.5(+/-1){ }^{\circ} \mathrm{C}$ with $80-95 \%$ humidity by adding water to the bottom of the incubator on a daily basis. Allow the incubator to equilibrate for two days before using.

1. Select small feed cups $(5 \times 7.6 \mathrm{~cm})$ at least $2.5 \mathrm{~cm}$ deep to hold eggs in the incubator. Line the bottom and lower edges of the cup with two layers of paper towel so that the cup is padded, while still allowing the eggs to easily roll. Place these cups on the tilting shelves of the incubator. Note: Too much padding can inhibit rolling, which will prevent successful incubation due to embryonic adhesion to the shell interior.

3. Collect the eggs at two hours following the onset of the light cycle. Note: This minimizes discrepancies in stage development for a given incubation time due to parental incubation.

1. Pick up eggs gently with thumb and forefinger at the tip and base along the length of the egg. Use a dull, soft 4B graphite pencil to label the date laid and time collected from the nest. Note: The softer 4B pencil reduces the risk of the pencil breaking the fragile eggshell.

2. 1.3.2) Place $1-5$ labeled eggs in each cup in the incubator so that eggs can freely roll to prevent embryonic adhesion to the interior of the shell. Note: Placing more than 5 eggs in a cup may prevent rolling of the individual eggs and reduce viability of embryos.

\section{Removal of Embryo from Egg}

1. To prepare for dissection, assemble the following materials: a clean scalpel, fine tipped forceps, and two extra fine tipped forceps. Place 10 $x 10 \mathrm{~cm}$ weigh paper on the base of the dissecting scope to provide a clean, non-absorbent surface for dissection. Note: The weigh paper is crucial because it allows easy manipulation of the yolk and is the best surface for cutting the delicate membranes with a scalpel.

2. Prepare an aliquot of phosphate buffer saline (1X PBS) in a $50 \mathrm{ml}$ polypropylene tube, and acquire at least three transfer pipettes and a small waste bucket. If fixing the embryos, prepare an aliquot of $4 \%$ paraformaldehyde (PFA).Caution: PFA vapors are toxic, and all steps involving PFA should be done inside a fume hood to minimize exposure. If flash freezing the embryos, obtain liquid nitrogen and keep it near the dissecting scope. Sterility is not an issue for this dissection protocol, but ensure all materials are clean.

3. Remove the egg from the incubator at the time point needed for the desired stage as described in the zebra finch staging guide ${ }^{22}$. Note: Remove eggs after $36 \mathrm{hr}$ of incubation to dissect stage 6 embryos (Figure 4A) ${ }^{22}$ and remove eggs after 56 hr of incubation to dissect stage 12 embryos (Figure 3A) ${ }^{22}$.

4. Using a fiber optic illuminator lamp, candle the egg by holding it along its vertical axis and shine light through the egg to illuminate the interior. Place the tip of the light behind the egg and locate the yolk and the developing embryo.

1. Position the scalpel on the side opposite the yolk and cut along the egg from tip to base with faint pressure.

5. Remove the contents of the egg by carefully applying pressure on the tip and base of the egg with the thumb and the forefinger, or by gently prying apart the eggshell along the cut with forceps. Open the egg directly above the weigh paper so that the yolk gently rolls out.

6. Examine the yolk for the white zone of junction, which is visible as a faint white ring encircling embryonic development and orient the yolk using extra fine tipped forceps so that the embryo is located in the center.

Note: If a faint white circle on the surface of the yolk is not observed, take the fine forceps and gently roll the yolk over until the embryo is on top of the yolk. Note: For stages $1-9^{22}$, the embryonic disk is less than $6 \mathrm{~mm}$ in diameter and is visible as a faint, slightly opaque disk on the surface of the yolk - see Figure 1 for reference. For stages 10 and older ${ }^{22}$, blood pools allow improved visibility of the embryo, but take care to avoid puncturing the yolk sac, as this makes locating the embryo difficult.

\section{Separation of Embryo from Extra-embryonic Tissue}

1. Puncture the edges of the yolk to relieve pressure (Figure 1B), and make single cuts across the length of the yolk diameter alongside the embryonic disk. Repeat making these diagonal lines until the section of the yolk containing the embryo has been successfully separated (Figure 1B). Note: This step allows the yolk mass to remain intact, but the reduced pressure on the surface of the yolk allows for more precision when cutting around the embryo, preventing damage to embryonic structures.

2. Remove the edges of the yolk with a transfer pipette. Note: Remove as much yolk as possible, but leave a small amount so that the embryo does not adhere to the dry surface of the weigh paper and tear.

3. Wash the embryonic disk by dispensing $1 \mathrm{X}$ PBS at a $45^{\circ}$ angle towards the bottom of the embryo. Place the tip of the transfer pipette next to-not above- the embryonic disk. If additional yolk needs to be removed, separate any yolk with the scalpel on the weigh paper before transferring the embryo to the Petri dish. Note: This method removes the embryo from the surface of the weigh paper.

4. Transfer the embryo using a transfer pipette along with a minimal volume of $1 X$ PBS to a small, plastic Petri dish. Wash the embryo by adding more 1X PBS into the Petri dish and dripping 1X PBS near, but not directly onto, the embryo. Swirl the Petri dish to wash and remove residual yolk, tilt the Petri dish, and remove waste $1 \mathrm{X}$ PBS with the transfer pipette.

NOTE: When the 1X PBS is removed, the embryo typically does not adhere to the bottom of the Petri dish because the plastic surface is slippery with residual $1 \mathrm{X}$ PBS. However, more $1 \mathrm{X}$ PBS can be added if the embryo sticks to the dish. If fixing the embryo, follow steps 3.5 and 3.6. If flash freezing the embryo, skip to step 3.8 immediately.

5. If fixing the embryo for in situ hybridization, immediately add 4\% PFA to the Petri dish to submerge the embryo. Drip 4\% PFA directly onto the top of the embryo in order to flatten it; this prevents the embryo from curling. Fix the embryo in $4 \%$ PFA at $4{ }^{\circ} \mathrm{C}$ for $12 \mathrm{hr}$.

1. After fixation, dehydrate embryos in graded methanol $(\mathrm{MeOH})$ solutions and store at $-20^{\circ} \mathrm{C}$ in $100 \% \mathrm{MeOH}$. 
6. If the embryo is between stages $1-8^{22}$, remove the vitelline membrane adhered to the embryo. Note: This step can be done during or after fixation. Embryos younger than stage $8^{22}$ are not easily visualized because they adhere to the vitelline membrane, obscuring key structures as seen in Figure $2 \mathrm{~A}$

1. Grip the edge of the membrane that extends beyond the zone of junction with extra fine forceps. Carefully flip the embryo over multiple times to wash away residual yolk granules and to loosen the adherence of the embryonic disk to the vitelline membrane. Note: Do not touch the center of the embryo, as this will damage embryonic structures.

2. If a gap does not appear between the zone of junction and the vitelline membrane, gently scratch the zone of junction with the extra fine tipped forceps to loosen it from the vitelline membrane. Grip the vitelline membrane with the extra fine tipped forceps and gently pull it away from the embryo. If necessary, gently pull the embryo away from the vitelline membrane by gripping the peripheral edge of the embryonic disk at the zone of junction.

3. Discard the vitelline membrane in biohazard 1 waste (biosafety level 1) after removal.

7. When conducting an ISH assay on young embryonic stages, follow standard whole mount ISH protocols for chick embryos ${ }^{24,25}$, but consider the following suggestions.

1. To reduce damage to the embryos during the ISH procedure, use a single $5 \mathrm{ml}$ glass vial with a screw cap for each embryo. Only fill vials with $2-3 \mathrm{ml}$ of solution, ensuring that the embryos are fully submerged. Nutate vials vertically by placing $5 \mathrm{ml}$ vials into a styrofoam rack (or any rack that will keep the vials secure) that is secured to a nutator. Note: This precaution prevents the embryo from being torn, which can occur when it comes into contact with the lid of the vial during horizontal nutation.

2. For embryos stage $0-6^{22}$, treat with $5 \mu \mathrm{g} / \mathrm{ml}$ proteinase $\mathrm{K}$ in $1 \mathrm{X}$ PTw for $5 \mathrm{~min}$ at room temperature. Treat embryos staged $7-12^{22}$ with $10 \mu \mathrm{g} / \mathrm{ml}$ proteinase $\mathrm{K}$ in $1 \mathrm{X} \mathrm{PTw}$ for $10 \mathrm{~min}$ at $37^{\circ} \mathrm{C}$ to reduce background staining.

3. To produce a sufficient color reaction to detect low levels of gene expression in stages $1-10^{22}$, incubate with $10 \mu \mathrm{g} / \mathrm{ml} \mathrm{probe}$ concentration for $12 \mathrm{hr}$. For older stages, incubate with $1 \mu \mathrm{g} / \mathrm{ml}$ probe concentration at $60^{\circ} \mathrm{C}$.

8. If flash-freezing the embryo, quickly add $2-3 \mathrm{ml} 1 \mathrm{X}$ PBS to the Petri dish following dissection and tilt the Petri dish to separate the embryo from the yolk granules. Remove liquid and repeat 2 - 3 times to remove all yolk. Using fine tipped forceps, transfer embryo to a pre-labeled microcentrifuge tube and flash freeze in liquid nitrogen before storing at $-80^{\circ} \mathrm{C}$.

\section{EdU Cell Proliferation Assay. Incorporation and Detection of EdU in Zebra Finch Embryos.}

1. Candle the egg using a fiber optic illuminator lamp to locate the embryo or yolk.

2. Mark the side of the egg opposite to the location of the embryo or yolk within the egg to ensure that the embryo is not damaged during the microinjection process.

3. Line a $60 \mathrm{~mm}$ plastic dish with modeling clay and mold it in the shape of a bowl to hold the egg in the desired orientation. The marked spot should be oriented to allow insertion of the microinjection needle using the micromanipulator. Note: The clay will stabilize the egg during microinjection and throughout the incubation in step 4.11.

4. Pull two glass capillary microinjection needles. Blunt one needle to poke a hole into the egg at the marked spot. Prepare the second microinjection needle for injection by backloading it with mineral oil and inserting it into the microinjector.

5. Set the volume of solution released per injection to $59.8 \mathrm{nl}$ on the microinjector.

6. Load the microinjection needle with the $10 \mathrm{mM}$ stock EdU solution.

7. Create a hole in the shell on the marked spot with the blunted glass capillary, taking care to not shatter the shell or drop pieces of the shell into the egg cavity. Orient the egg so that the hole is at a $45^{\circ}$ angle, allowing the microinjection needle to be inserted directly through the cavity to the yolk or embryo.

8. Using the micromanipulator, insert the loaded needle approximately $1.0 \mathrm{~cm}$ into the cavity.

9. Inject the desired amount of the EdU solution directly onto the developing embryo or yolk. Note: A maximum of $478 \mathrm{nl}$ of EdU can be injected without resulting in embryonic death.

10. Immediately wrap the egg and the clay holder with multiple layers of plastic wrap to cover the egg and the dish to prevent desiccation of the embryo during incubation. Tape the edges of the plastic wrap to the bottom of the dish to prevent the plastic from unwrapping during incubation. Note: The plastic wrap should only be removed immediately before dissection.

11. Allow the newly proliferating cells to incorporate the EdU by incubating the egg at $37^{\circ} \mathrm{C}$ until the desired stage is reached. After injection, do not return egg to tilting shelves in incubator. Place the plastic-wrapped clay lined dish on stable surface inside the incubator.

12. Remove the plastic wrap and dissect the embryo following the aforementioned protocol. Fix the embryo in $4 \%$ PFA and incubate 12 hr at $4{ }^{\circ} \mathrm{C}$ as described above. After fixation, wash with $100 \%$ ethanol $(\mathrm{EtOH})$ for $5 \mathrm{~min}$ and store in fresh $100 \% \mathrm{EtOH}$ at $-20^{\circ} \mathrm{C}$ until further analysis.

\section{EdU “Click" Reaction Protocol}

The following steps are all performed in glass vials.

1. Rehydrate embryos with successive 5 min washes of the following:

2. $\mathrm{EtOH}, 75 \% \mathrm{EtOH}$ and $25 \%$ sterile deionized distilled (sdd) water, $50 \% \mathrm{EtOH}$ and $50 \%$ sdd water, $25 \% \mathrm{EtOH}$ and $75 \% 1 \mathrm{XPTw}, 100 \% 1 \mathrm{X} \mathrm{Ptw}$

3. Wash three times in $100 \%$ 1X PTw for 10 min each.

4. Dilute the reaction buffer additive (Kit Component $F$ ) by combining 1 part of the $10 X$ stock buffer solution (10 $\mu$ l) to 9 parts sdd water ( $90 \mu \mathrm{l})$.

5. Prepare the reaction mix in a separate tube by mixing the following: $875 \mu \mathrm{l}$ X PBS, $20 \mu \mathrm{l} \mathrm{CuSO}{ }_{4}, 5 \mu \mathrm{l}$ azide, $100 \mu \mathrm{l}$ diluted reaction buffer additive. Note: Volume of the reaction mix can be scaled down. The reaction will work if the embryo is completely covered in the reaction mixture. Add the diluted reaction buffer additive (step 5.3) immediately before use. All subsequent steps are performed in the dark. Cover the embryos with foil to protect from the light.

6. Cover the vials with aluminum foil, and incubate them vertically at room temperature for two hours by placing them in a styrofoam rack attached to a nutator. 
7. Wash the embryos 3 times in fresh $1 X$ PBS for 10 min each.

8. Incubate the embryos in fresh $4 \%$ PFA overnight at $4{ }^{\circ} \mathrm{C}$.

9. Wash 3 times in $1 \mathrm{X}$ PBS for 10 min each and store in fresh $1 \mathrm{X} P B S$ at $4{ }^{\circ} \mathrm{C}$. Cover the embryos with foil until further analysis.

\section{Representative Results}

The steps diagrammed in Figure 1 indicate the appearance of the embryo while attached to the vitelline membrane (A) and demonstrate the proper method to separate the embryo from the yolk (B). The embryo can be identified by the zone of junction, which is much lighter than the vitelline membrane. The embryo itself is often difficult to distinguish until the yolk is cut away. Once the embryo is dissected from the egg, it can be fixed or flash frozen for future use. If an in situ hybridization is planned for the dissected embryo, it is necessary to remove the vitelline membrane that is adhered to the embryo via the zone of junction. Figure 2 illustrates the improved visibility of the embryo once this membrane is removed $(C)$, and the proper way to peel the vitelline membrane (A, B). After dissection and fixation, whole mount in situ hybridization was performed as seen in Figure 3 (A, A', B, B') and Figure 4 (A, A', B, B') and Figure 5 (A, B, C) to detect differences in orthodenticlehomeobox 2 (Otx2) expression in embryos developmentally exposed to low doses of methylmercury. Figure 5 shows sense probe results, demonstrating lack of background. In Figure 4, despite being dissected from the egg at the same time point, the embryo developmentally exposed to methylmercury $(\mathrm{MeHg})$ progressed to stage $5^{22}\left(\mathrm{~B}, \mathrm{~B}^{\prime}\right)$, while the control embryo developed to stage $6^{22}\left(\mathrm{~A}, \mathrm{~A}^{\prime}\right)$. The group of embryos dissected and shown in Figure 4 were collected from the nest and taken from the incubator at the same times. Although some natural variation is present in development, based on previous dissection data, it is unlikely that temperature fluctuations in the incubator would cause only the $2.4 \mathrm{ppm}$ methylmercury embryos to be developmentally delayed. The differences in stages indicate changes in cell proliferation in embryos developmentally exposed to methylmercury.

Before dissection, EdU was injected into a day 2 egg and allowed to incubate overnight. After dissection and fixation of the stage $16^{22}$ embryo, EdU was visualized using "click" chemistry, allowing detection of proliferating cells as seen in Figure 6 (A, B, C). It is important to carefully monitor time points when placing eggs in the incubator and during dissections, as exposure to methylmercury or performing the EdU assay may disrupt developmental progression. The earliest injection was performed on day 0 , which was the day of collection as specified in step 1.3. This embryo was dissected approximately 38 hours later (stage $7^{22}$ ). The survival rate was found to be approximately $90 \%$ (same rate as control embryos) as long as the injection amount was under $478 \mathrm{nl}$.

This dissection methodology also allows for high quality RNA extraction. After dissecting stage $16^{22}$ embryos, an RNA extraction was performed according to manufacturer's protocol with no optimization required, as seen in Figure 7 . The removal of the vitelline membrane was unnecessary for RNA extraction and later qRT-PCR applications.

Note: All embryo figures are oriented so that the anterior and posterior regions are at the top and bottom of the images, respectively.
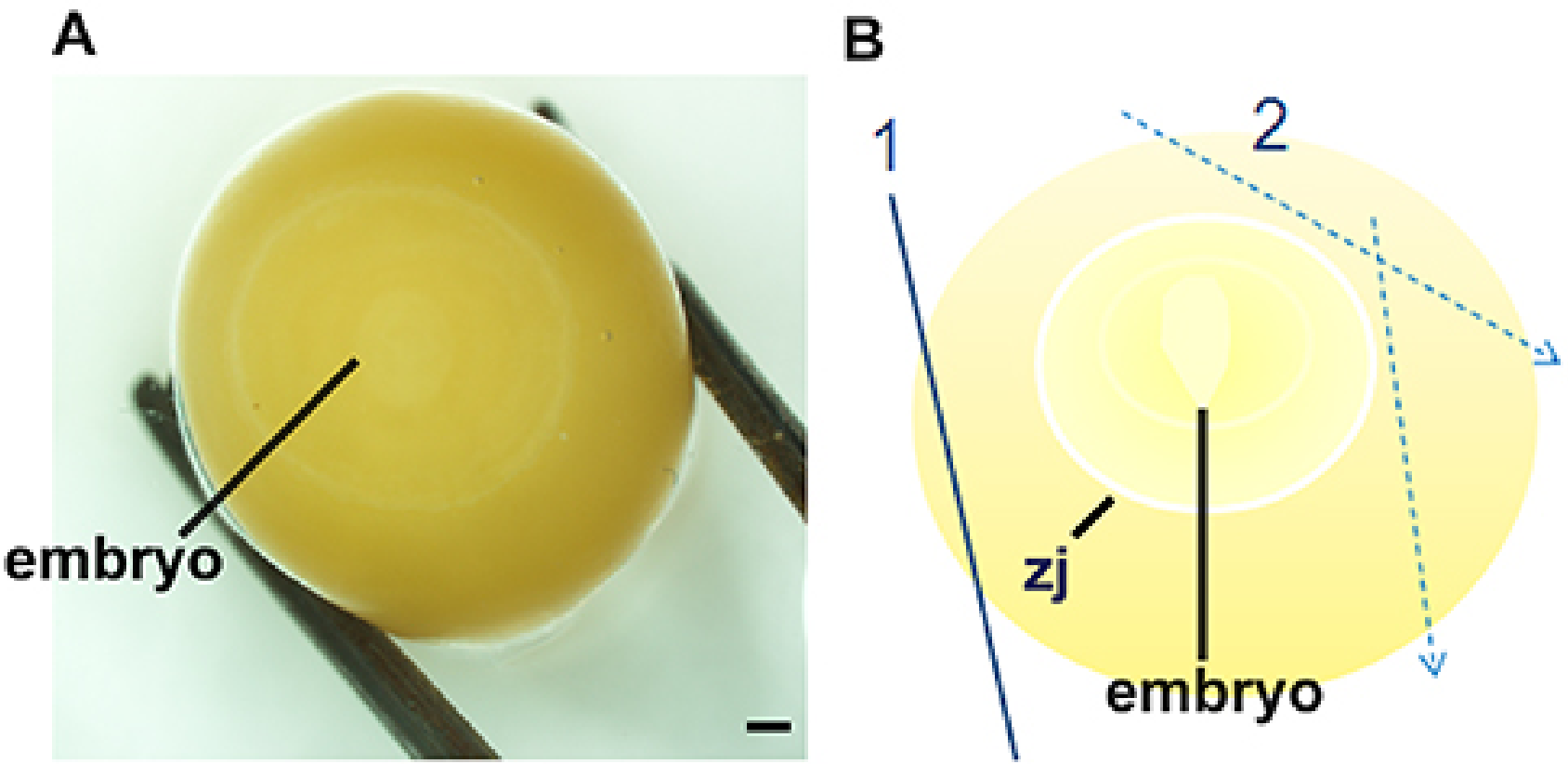

Figure 1. Procedure for locating and dissecting zebra finch embryos, stages $1-10^{22}$. Locate embryo by gently rolling the yolk until the faint white disk is apparent (A). Once the embryo is located at the center of the yolk, the yolk is dissected in a stepwise fashion (B) where the first cut relieves pressure of the vitelline membrane (1) and subsequent cuts (2) border the zone of junction (zj) which is adhered to the vitelline membrane. Scale bars represent $1 \mathrm{~mm}$. 

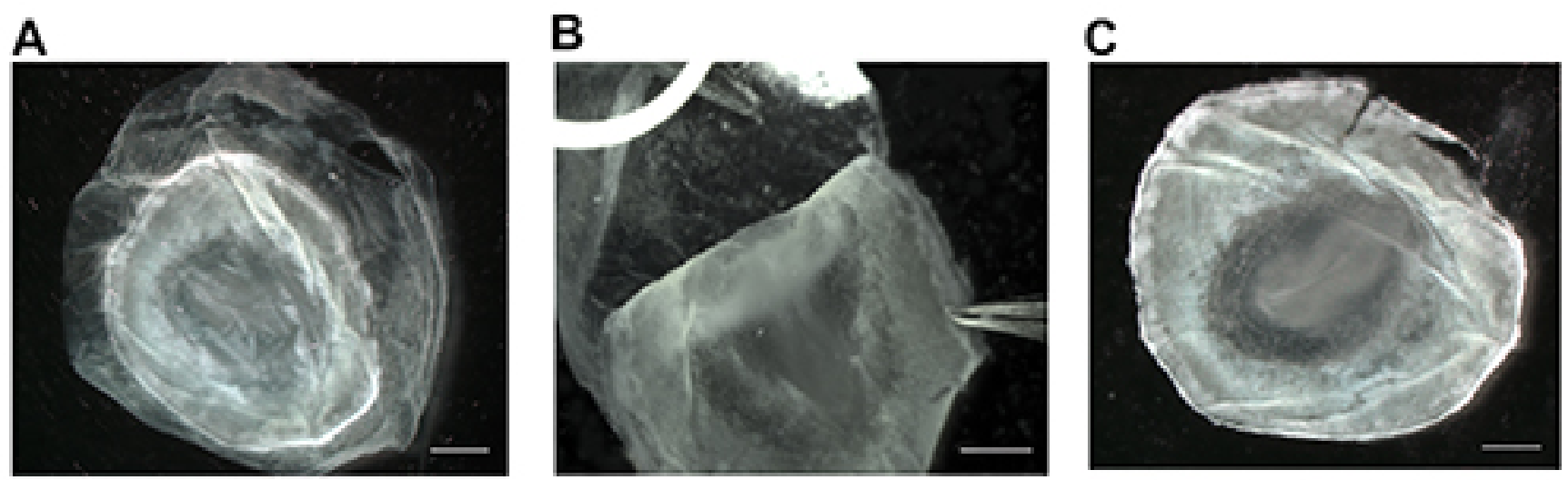

Figure 2. Removal of vitelline membrane and visibility of embryonic structures. Following removal of the embryo from the yolk, place embryo in a Petri dish containing 4\% PFA. If an in situ hybridization needs to be performed, visibility of the embryonic structures is essential and can be achieved by removing the vitelline membrane (A). Grip the vitelline membrane with extra fine tipped forceps and gently peel it away from the embryo by handling the embryo directly at the outermost edge, if necessary (B). Vitelline membrane removal increases clarity of embryonic structures, and allows embryos to be imaged or processed with in situ hybridization (C). Scale bars represent $1 \mathrm{~mm}$. Please click here to view a larger version of this figure.
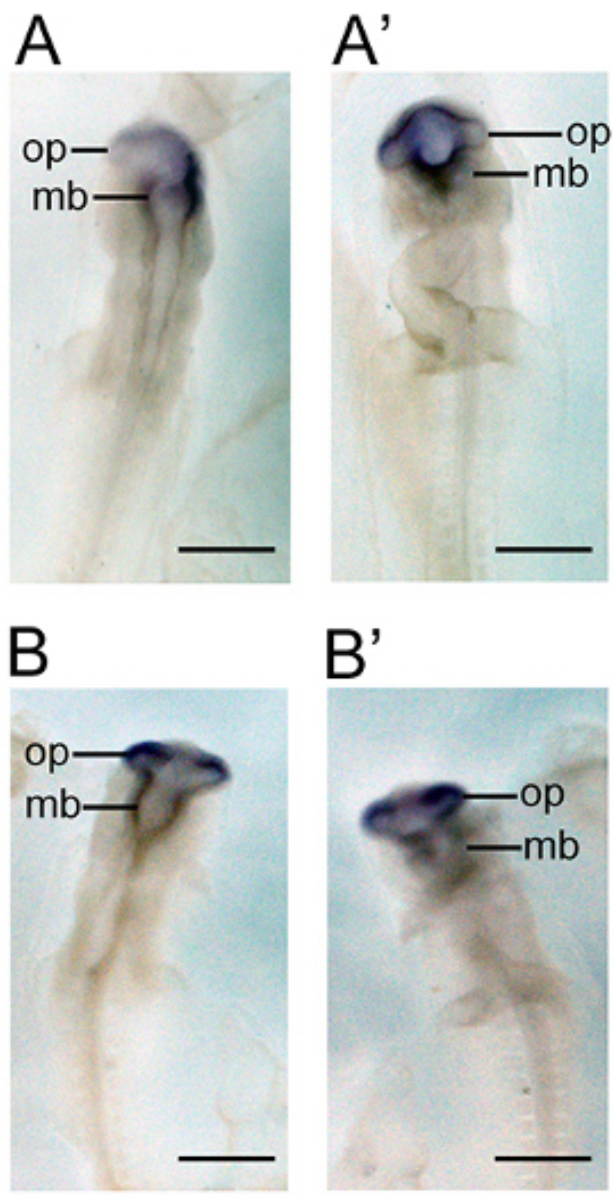

Figure 3. Whole mount in situ hybridization performed on zebra finch embryos developmentally exposed to methylmercury.

Expression patterns of orthodenticlehomeobox 2 (Otx2) were characterized in embryos exposed to 0.0 ppm methylmercury (A, A') and 2.4 ppm methylmercury (B, B') via parental diet. The dorsal $(A)$ and ventral $\left(A^{\prime}\right)$ expression of Otx2 is visible throughout the midbrain and optic vesicles during stage $12^{22}$. The treatment group embryos were dissected at the same time point, but were developmentally delayed as seen in the dorsal $(B)$ and ventral $\left(B^{\prime}\right)$ view of head structures, which are characteristic of stage $11^{22}$. Abbreviations: mb, midbrain; op, optic vesicle. Scale bars represent $1 \mathrm{~mm}$. 
A

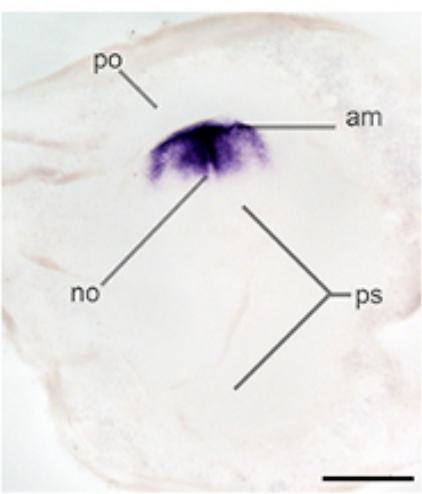

B

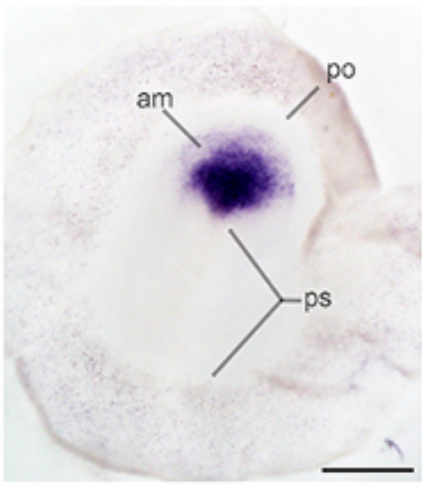

$A^{\prime}$

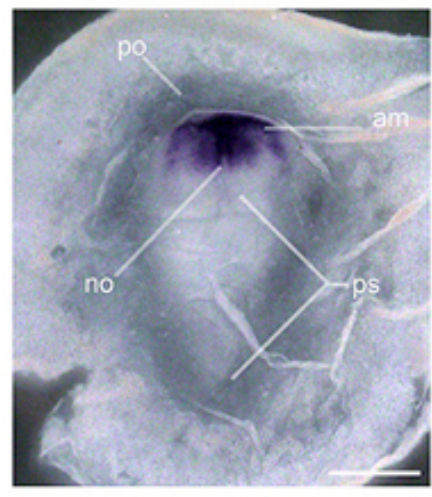

$B^{\prime}$

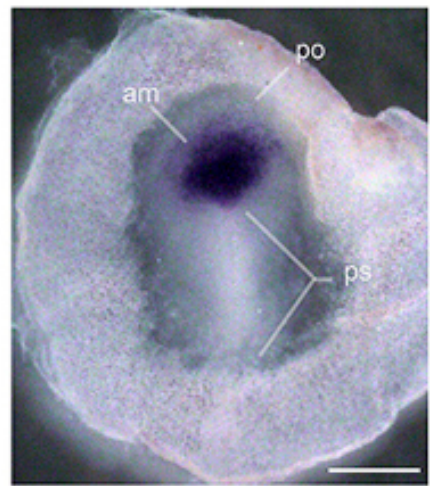

Figure 4. Whole mount in situ hybridization performed on zebra finch embryos developmentally exposed to methylmercury. Expression patterns of orthodenticlehomeobox 2 (Otx2) were characterized in stage $6^{22}$ embryos exposed to 0.0 ppm methylmercury (A, A') and stage $5^{22}$ embryos exposed to 2.4 ppm methylmercury (B, B') via parental diet. Abbreviations: am, anterior margin of mesoderm; no, notochord, notochord mesoderm; po, proamnion, anterior blastopore; ps, primitive streak ${ }^{22}$. Scale bars represent $1 \mathrm{~mm}$.
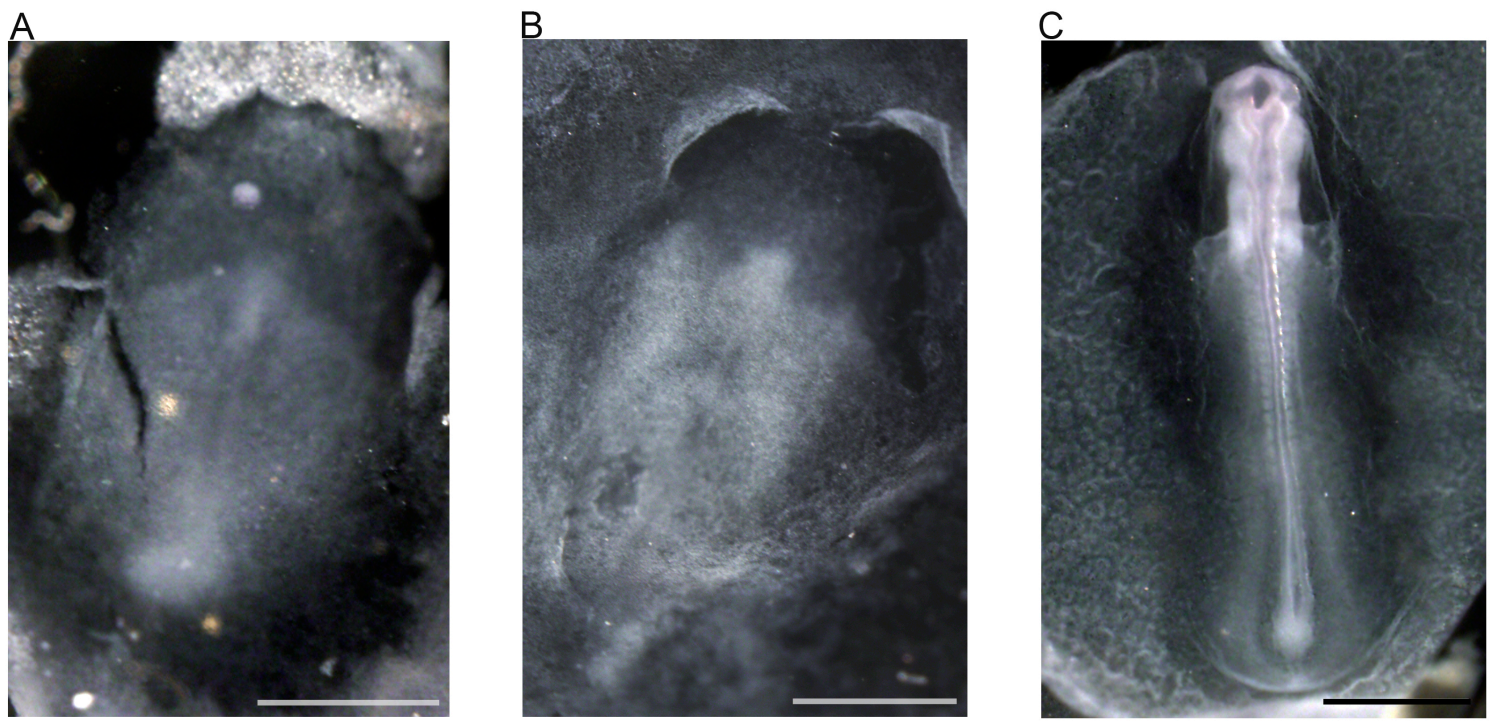

Figure 5. Whole mount in situ hybridization performed on zebra finch embryos using sense probe. (A) Stage $5^{22}$ embryo. (B) Early stage $6^{22}$ embryo. (C) Stage $11^{22}$ embryo. Scale bars represent $1 \mathrm{~mm}$. 

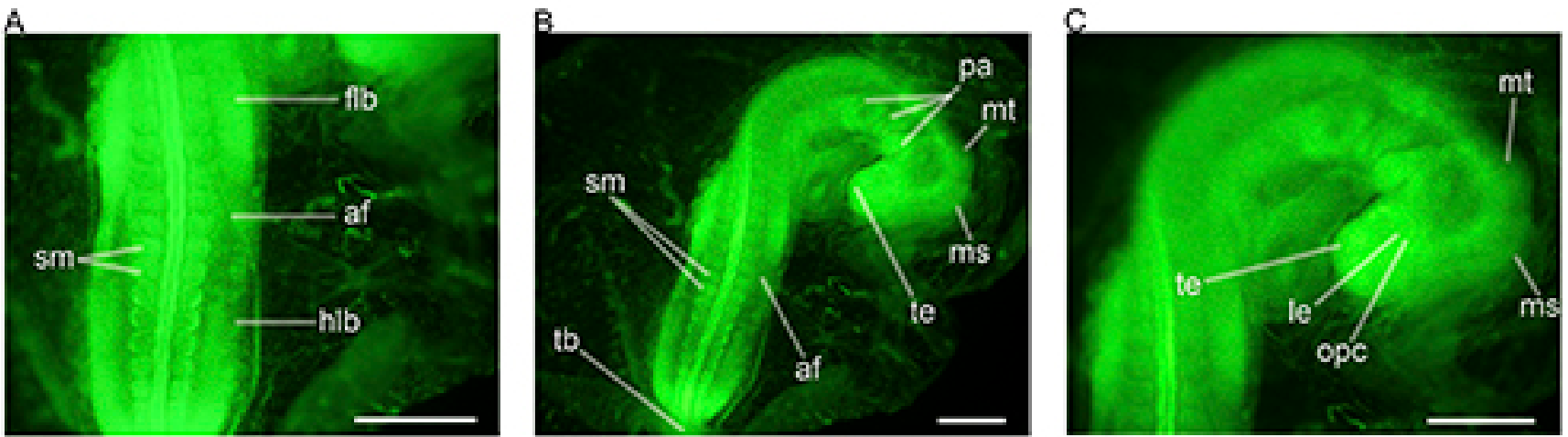

Figure 6.EdU incorporation and detection in zebra finch embryos. EdU "click" chemistry was used to detect proliferating cells in a stage $16^{22}$ embryo $(A, B, C)$. EdU is incorporated into the DNA in the place of thymidine ${ }^{26,27}$ and is detected using click chemistry ${ }^{27}$. Proliferation is clearly visible in the lateral edges of the somites, and the tailbud. Panel A shows proliferation occurring exclusively in the posterior of the embryo and also shows individual proliferative cells. Panel B shows the proliferative locations in the whole embryo. Panel $\mathbf{C}$ shows the anterior region, and shows the highly proliferative telencephalon (te) in greater detail. Abbreviations: af, amniotic fold; flb, forelimb bud; hlb, hindlimb bud; le, lens vesicle; ms, mesencephalon; mt, metencephalon; opc, optic cup; pa, pharyngeal arch; sm, somite mesoderm; tb, tailbud; te, telencephalon. Scale bars represent $1 \mathrm{~mm}$. Please click here to view a larger version of this figure.

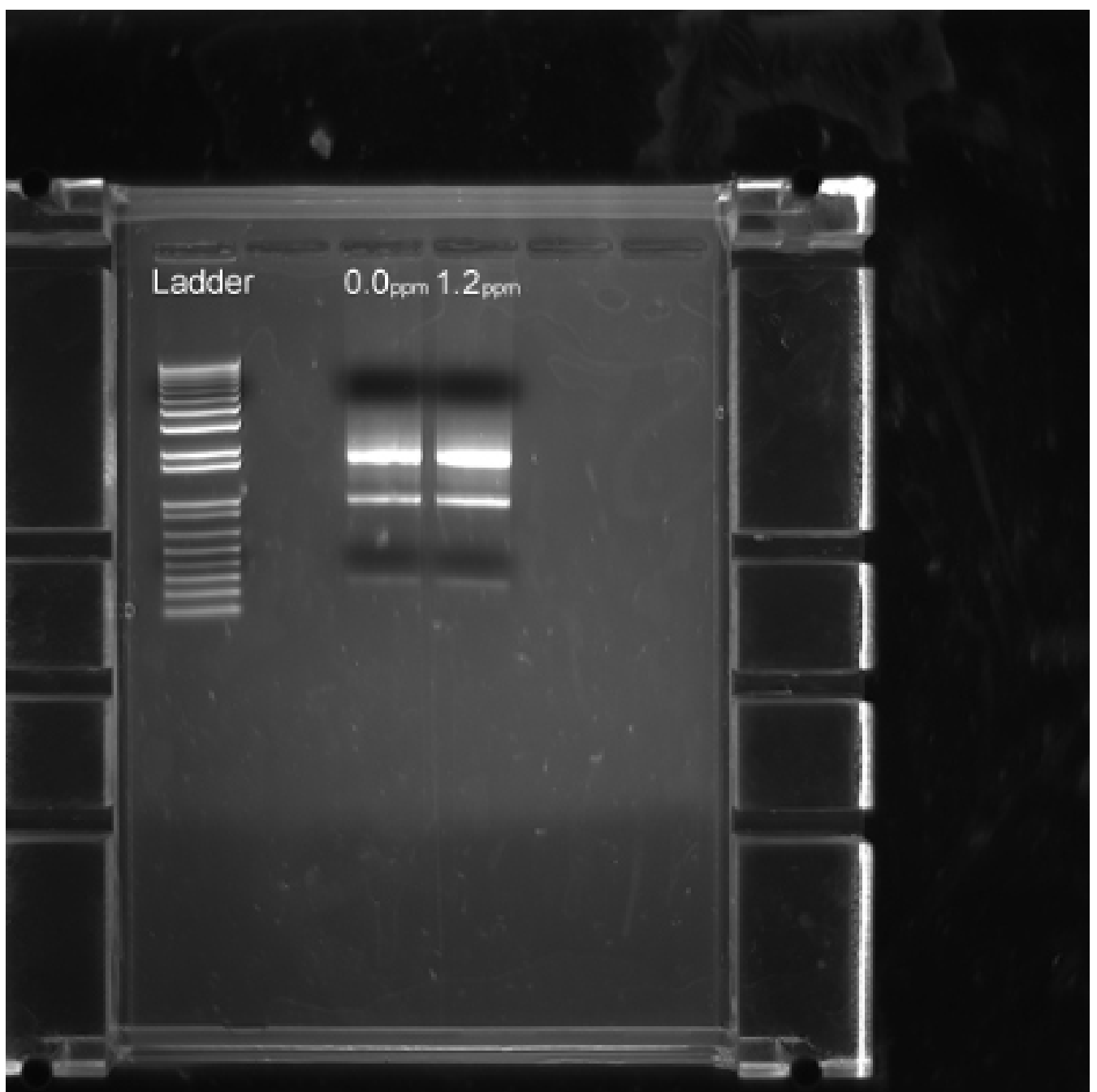

Figure 7. Quality of RNA extracted from dissected zebra finch embryos. Control $(0.0 \mathrm{ppm})$ and 1.2 ppm methylmercury embryos were dissected and flash frozen as described in Step 3.8. Each lane shows RNA extracted from two homogenized embryos from each treatment group. 


\section{Discussion}

Recent development of an embryological staging guide ${ }^{22}$ and genome annotation make the zebra finch a desirable model organism for developmental studies. However, the small size and fragility of the zebra finch embryos, which range from 3 to $7 \mathrm{~mm}$ in stages $1-10^{22}$, can make dissections difficult ${ }^{11,14}$. Locating and cleanly removing embryos from the surface of the yolk can be challenging. This protocol provides sufficient detail to perform the procedure with ease. This protocol demonstrates the critical steps that are not typically known, but are necessary to ensure a successful dissection. For example, it is essential to leave a small layer of yolk between the embryo and sheet of weigh paper to preclude sticking.

Both the identification and removal of the embryo can be difficult. To troubleshoot identifying the embryo on the surface of the yolk, shine light directly above the yolk after it has been removed from the egg, and look at the yolk at a $45^{\circ}$ angle to find the embryo. Once the embryo is located, cut the yolk on weigh paper taking care to not tear the embryo.

If further applications include imaging for anatomical differences, in situ hybridization, or cell proliferation assays, it is important to remove the vitelline membrane in stage $1-8^{22}$ for better visualization of structures. If experiencing difficulties when removing the yolk or the vitelline membrane in early stages, fix the embryo in $4 \%$ PFA before washing it in 1X PBS to reduce embryonic fragility. By first removing the vitelline membrane during dissection as described, structures are clearly visible and intact in zebra finch embryos after performing an in situ hybridization.

A limitation of EdU is that administration of dosage volumes over $478 \mathrm{nl}$ leads to embryonic fatality. However, the vast dosage range allows varying levels of proliferative cell tagging.

The "click" reaction used in this kit is the Copper(I)-Catalyzed-Alkyne-Azide-Cycloaddition (Cu(I)AAC). In this specific reaction, an alkynecontaining thymidine analogue molecule (EdU) is incorporated by actively dividing cells. The alkyne group in the EdU protrudes from the helical structure of the DNA, and is detected by exposure to an azide molecule conjugated to a green, fluorescent molecule which binds to the free alkyne group. The green fluorescence shows the newly proliferating cells in the embryo. The bio-orthogonality of the azide and the alkyne groups prevents non-specific staining because these reactive species are not naturally present in organisms. Also, because the DNA does not need to be denatured in order for the reaction to occur, further DNA-dependent analysis can be easily performed ${ }^{27}$.

An inherent limitation of this method is the small size and fragility of zebra finch embryos. Removing the vitelline membrane of embryos stages $1-15^{22}$ can result in damaging embryonic structures if not performed with caution. However, this protocol simplifies the dissection method, allowing investigators to use early embryonic stages to examine structural anomalies and gene expression that have not been previously studied in depth. This protocol opens the way for a plethora of cellular-molecular assays that will allow investigators to determine the developmental origins of adult phenotypes. For example, it will be possible to examine gene expression implicated in vocal learning under various environmental conditions or following pharmacological treatments at the earliest stages of development ${ }^{28,29,30,31}$. Although not demonstrated in this paper, this method potentially allows for other procedures such as radioactive in situ hybridization on zebra finch tissue sections and electroporation/in ovosurgery ${ }^{32,33,34}$. Given that the zebra finch has been established as an important model organism within a vast body of literature, such studies provide untapped opportunities to link developmental mechanisms with adult physiology and behavior, in particular the development of language l,9. $^{7,9}$.

\section{Disclosures}

The authors have nothing to disclose.

\section{Acknowledgements}

The authors thank their funding sources, Howard Hughes Medical Institute Undergraduate Science Education Program to the College of William and Mary; Grant sponsor: NIH (M.S.S); Grant number: R15NS067566. They also acknowledge support from the College of William and Mary, Department of Biology and College of Arts and Sciences for assistance with animal care.

\section{References}

1. Eng, M. L., Elliott, J. E., MacDougall-Shackleton, S. A., Letcher, R. J., \& Williams, T. D. Early exposure to 2,2',4,4',5-pentabromodiphenyl ether (BDE-99) affects mating behavior of zebra finches. Toxicol Sci. 127, 269-276, doi:10.1093/toxsci/kfs076 (2012).

2. Winter, V., Elliott, J. E., Letcher, R. J., \& Williams, T. D. Validation of an egg-injection method for embryotoxicity studies in a small, model songbird, the zebra finch (Taeniopygia guttata). Chemosphere. 90, 125-131, doi:10.1016/j.chemosphere.2012.08.017 (2013).

3. Maney, D. L., \& Goodson, J. L. Neurogenomic mechanisms of aggression in songbirds. Adv Genet. 75, 83-119, doi:10.1016/ B978-0-12-380858-5.00002-2 (2011).

4. Kirn, J. R. The relationship of neurogenesis and growth of brain regions to song learning. Brain and Language. 115, 29-44, doi:10.1016/ j.bandl.2009.09.006 (2010).

5. Fisher, S. E., \& Scharff, C. FOXP2 as a molecular window into speech and language. Trends in Genetics. 25, 166-167, doi:10.1016/ j.tig.2009.03.002 (2009).

6. Tokarev, K., Tiunova, A., Scharff, C., \& Anokhin, K. Food for Song: Expression of C-Fos and ZENK in the Zebra Finch Song Nuclei during Food Aversion Learning. PLoS One. 6, doi:10.1371/journal.pone.0021157 (2011).

7. Vargha-Khadem, F., Gadian, D. G., Copp, A., \& Mishkin, M. FOXP2 and the neuroanatomy of speech and language. Nat Rev Neurosci. 6, 131-138 (2005). 
8. Schulz, S. B., Haesler, S., Scharff, C., \& Rochefort, C. Knockdown of FoxP2 alters spine density in Area X of the zebra finch. Genes, Brain and Behavior. 9, 732-740, doi:10.1111/j.1601-183X.2010.00607.x (2010).

9. Jarvis, E. D. et al. Avian brains and a new understanding of vertebrate brain evolution. Nat Rev Neurosci. 6, 151-159, doi:10.1038/nrn1606 (2005).

10. Brainard, M. S., \& Doupe, A. J. Translating birdsong: songbirds as a model for basic applied medical research. Annu Rev Neurosci. 36, 489-517, doi:10.1146/annurev-neuro-060909-152826 (2013).

11. Mayer, U., Watanabe, S., \& Bischof, H. J. Spatial memory and the avian hippocampus: Research in zebra finches. J Physiol Paris. doi:10.1016/j.jphysparis.2012.05.002 (2012).

12. Warren, W. C. et al. The genome of a songbird. Nature. 464, 757-762, doi:10.1038/nature08819 (2010).

13. Agate, R. J., Scott, B., Haripal, B., Lois, C., \& Nottebohm, F. Transgenic songbirds offer an opportunity to develop a genetic model for vocal learning. Proceedings of the National Academy of Sciences. 106, 17963-17967, doi:10.1073/pnas.0909139106 (2009).

14. Birkhead, T. R. et al. Internal incubation and early hatching in brood parasitic birds. Proceedings of the Royal Society Biological Sciences. 278, 1019-1024, doi:10.1098/rspb.2010.1504 (2011).

15. Haesler, S. et al. FoxP2 expression in avian vocal learners and non-learners. J Neurosci. 24, 3164-3175 (2004).

16. Godsave, S. F., Lohmann, R., Vloet, R. P. M., \& Gahr, M. Androgen receptors in the embryonic zebra finch hindbrain suggest a function for maternal androgens in perihatchingsurvival. Journal of Comparative Neurology. 453, 57-70, doi:10.1002/cne.10391 (2002).

17. Stern, C. D. The chick; a great model system becomes even greater. Dev Cell. 8, 9-17 (2005).

18. Kuenzel, W. J., Medina, L., Csillag, A., Perkel, D. J., \& Reiner, A. The avian subpallium: new insights into structural and functional subdivisions occupying the lateral subpallial wall and their embryological origins. Brain Research. 1424, 67-101, doi:10.1016/ j.brainres.2011.09.037 (2011).

19. Tickle, C. The contribution of chicken embryology to the understanding of vertebrate limb development. Mech Dev. 121, 1019-1029 (2004).

20. Vergara, M. N., \& Canto-Soler, M. V. Rediscovering the chick embryo as a model to study retinal development. Neural Development. 7 , doi:10.1186/1749-8104-7-22 (2012).

21. Davey, M. G., \& Tickle, C. The chicken as a model for embryonic development. Cytogenet Genome Research. 117, 231-239, doi:10.1159/000103184 (2007)

22. Murray, J. R., Varian-Ramos, C. W., Welch, Z. S., \& Saha, M. S. Embryological staging of the zebra finch, Taeniopygia guttata. J Morphol., doi:10.1002/jmor.20165 (2013).

23. Hawkins, P., Morton, D. B., Cameron, D., Cuthill, I., Francis, R., Freire, R., Gosler, A., Healy, S., Hudson, A., Inglis, I., Jones, A., Kirkwood, J., Lawton, M., Monaghan, P., Sherwin, C., \& Townsend, P. Laboratory birds: refinements in husbandry and procedures. Fifth report of BVAAWF/ FRAME /RSPCA/UFAW Joint Working Group on Refinement. Lab Anim. doi: 10.1258/0023677011911967 (2001).

24. Pearse II, R. V., Esshaki, D., Tabin, C. J., \& Murray, M. M. Genome-wide expression analysis of intra- and extraarticular connective tissue. Journal of Orthopaedic Research. 27, 427-434, doi:10.1002/jor.20774 (2009).

25. Pizard, A. et al. Whole-mount in situ hybridization and detection of RNAs in vertebrate embryos and isolated organs. Current Protocols in Molecular Biology. 66, 14.19.11-14.19.24, doi:10.1002/0471142727.mb1409s66 (2004).

26. Warren, M., Puskarczyk, K., \& Chapman, S. C. Chick embryo proliferation studies using EdUlabeling. Developmental Dynamics. 238, 944-949, doi:10.1002/dvdy.21895 (2009).

27. Salic, A., \& Mitchison, T. J. A chemical method for fast and sensitive detection of DNA syntesis in vivo. Proceedings of the National Academy of Sciences U S A. 105, 2415-2420, doi:10.1073/pnas.0712168105 (2008).

28. Hoogesteijn, A. L., DeVoogd, T. J., Quimby, F. W., De Caprio, T., \& Kollias, G. V. Reproductive impairment in zebra finches (Taeinopygia guttata). Environmental Toxicology and Chemistry. 24, 219-223, doi:10.1897/03-632R.1 (2005).

29. Winter, V., Williams, T. D., \& Elliott, J. E. A three-generational study of In ovoexposure to PBDE-99 in the zebra finch. Environmental Toxicology and Chemistry. 32, 562-568, doi:10.1002/etc.2102 (2013).

30. Kitulagodage, M., Buttemer, W. A., \& Astheimer, L. B. Adverse effects of fipronil on avian reproduction and development: maternal transfer of fipronil to eggs in zebra finch Taeinopygia guttata and in ovo exposure in chickens Gallus domesticus. Ecotoxicology. 20,653-660, doi:10.1007/s10646-011-0605-5 (2011).

31. Hallinger, K. K., Zabransky, D. J., Kazmer, K. A., \& Cristol, D. A. Birdsong differs between mercury-polluted and reference sites. The Auk. 127, 156-161, doi:10.1525/auk.2009.09058 (2010).

32. Chen, C. C., Wada, K., \& Jarvis, E. D. Radioactive in situ Hybridization for Detecting Diverse Gene Expression Patterns in Tissue. Journal of Visualized Experiments. 3764, doi:10.3791/3764 (2012).

33. Chen, C. C., Balaban, E., \& Jarvis, E. D. Interspecies Avian Brain Chimeras Reveal That Large Brain Size Differences Are Influenced by Cell-Interdependent Processes. PLoS One. 7, doi:10.1371/journal.pone.0042477 (2012).

34. Chen, C. C., Winkler, C. M., Pfenning, A. R., \& Jarvis, E. D. Molecular profiling of the developing avian telencephalon:Regional timing and brain subdivision continuities. The Journal of Comparative Neurology. 521, 3666-3701, doi:10.1002/cne.23406 (2013). 